\title{
155. A Characterisation of Regular Semi-group
}

\author{
By Kiyoshi Iséki
}

(Comm. by K. Kunugr, M.J.A., Nov. 12, 1956)

The concept of regular ring was introduced by J. v. Neumann [3]. Recently, L. Kovács [1] has given an interesting characterisation of regular ring. On the other hand, some writers studied regular semi-groups. In this short Note, we shall give a characterisation for regular semi-group which is similar to Theorem 1 of L. Kovács [1].

Following W. D. Munn and R. Penrose [2], we define a regular semi-group. A semi-group is said to be regular if for any given element $a$ of $S$ there is at least one element $x$ of $S$ such that $a x a=a$. A non empty subset $L$ of $S$ is said to be a left ideal if $S L \subset L$. Similarly we define right ideals. Then we have the following

Theorem 1. Any semi-group $S$ is regular if and only if

$$
A B=A \frown B
$$

for every right ideal $A$ and every left ideal $B$ of $S$.

Proof. Let $S$ be a regular semi-group, and let $a \in A \frown B$, then there is an element $x$ such that $a x a=a$. Since $B$ is a left ideal, $x a \in B$. Therefore $a=a(x a) \in A B$. This shows $A B \supset A \frown B$. Clearly $A B \subset A \frown B$. Hence $A B=A \frown B$.

To prove the converse, let $a$ be an element of $S$. Then $\{a x \mid x \in S\} \smile a$ is the right ideal $(a)$ of $S$ generated by $a$. By the hypothesis,

$$
(a)=(a) \frown S=(a) S=a S \text {. }
$$

Therefore, we have $a \in a S$. Similarly $a \in S a$. Hence

$$
a \in a R \frown R a=a R^{2} a,
$$

and there is an element $x$ such that $a=a x a$.

Now, let us suppose that a given regular semi-group $S$ is commutative, then, by Theorem 1 , any ideal $A$ in $S$ is idempotent, i.e. $A^{2}=A$. Conversely, suppose that every ideal in a commutative semigroup $S$ is idempotent. If $A$ and $B$ are ideals in $S$, then we have $A \frown B=(A \frown B)^{2}=(A \frown B)(A \frown B) \subset A B$. On the other hand, $A \frown B \supset A B$. Hence $A \frown B=A B$. By Theorem $1, S$ is regular, therefore we have the following

Theorem 2. A commutative semi-group is regular if and only if every ideal is idempotent.

From Theorem 2, it is easily seen that there is no non-zero nilpotent element in a commutative regular semi-group with 0 .

Corollary. Any commutative regular semi-group with 0 does not 
contain non-zero nilpotent element.

Corollary follows from the identity $a^{2} x=a$ also.

\section{References}

[1] L. Kovács: A note on regular rings, Publ. Math., Debrecen, 4, 465-468 (1956).

[2] W. D. Munn and R. Penrose: A note on inverse semigroups, Proc. Cambridge Phil. Soc., 51, 396-399 (1955).

[3] J. v. Neumann: On regular rings, Proc. Nat. Acad. Sci. U.S. A., 22, 707-713 (1936). 